\title{
SIMULATION OF PEDESTRIAN AT INTERSECTION IN URBAN CONGESTED AREA
}

\author{
Dipika Gupta ${ }^{1}$, V.R. Patel ${ }^{2}$ \\ ${ }^{1}$ M.E Student, Civil Engineering Department, L.D.College of Engineering, Ahmedabad-380015, Gujarat, India \\ ${ }^{2}$ Associate Professor, Civil Engineering Department, Government Engineering College, Patan, Gujarat, India
}

\begin{abstract}
At present, with limitation of land space lots of dense urban area facing pressure and problem related to traffic congestion. Vehicles and pedestrian are the prime road occupant in traffic. Walking is a sustainable mode of travel, and most journeys involve a walking component, whether the main portion of the trip is made by foot, car, or using public transport. People always tend to solve problem related to vehicular traffic, so there is need to simulate pedestrian also. For a proper simulation of urban traffic scenarios, besides cars other road users, namely pedestrians, have to be modeled. We will investigate to what extent it is possible to capture traffic effects imposed by simulated pedestrians by providing Skywalk and then perform without simulations that is real situation. As a developing State, mixed flow of the Ahmedabad urban road traffic is most obvious characteristic at pedestrian crossing where conflicts between the pedestrians and motor vehicle occur frequently. In the pedestrian crossing, pedestrians coming from both sides and the motor vehicles coming from different direction may have conflicts, so delay or accidents occur sometimes. Conflicts between the pedestrians and vehicles have negative effect on the traffic efficiency of the urban road network and the service level of the traffic. This work presents a real scenario at Intersection for pedestrian without indulging Pedestrians movement in the main traffic by using computer application (i.e.-VISSIM software), comparing the real field data with the simulated one. Therefore no delay will occur to the Pedestrian, and this provide better mobility to pedestrians in Urban Areas.
\end{abstract}

Keywords: Pedestrian Delay1, Pedestrian Simulation 2, VISSIM 3, and Skywalk 4.

\section{INTRODUCTION}

Since the 1960s, skyway systems (also known as skywalk systems) have become an important feature in many cities around the world. Ahmedabad is the largest city and former capital of the Indian state of Gujarat. With growing population, congestion, pollution and related issues, that are facing mobility accessibility problems, and the governments have become more supply oriented and thus preoccupied in building more roads, flyovers that they have completely neglected the most ancient mode of transport. Walking is a universal phenomenon but generally not considered as a transport mode because it does not employ vehicles as modes. The term "Pedestrian" is used to recognize the fact that the approach to pedestrian pathway development must be as scientific and systematic as the techniques which are applied to highway design and development. Hence safety of pedestrian is a basic step to create safer city. In metro cities, more than one mode of public transport is available now-adays. Due to expansion of city boundary and urban sprawls, the single mode of transport is neither viable nor efficient as the spatial separation between commuters and work places has increased. Hence, public transport has become multi modal which combines two or more modes to provide comfort, rapid and environmentally compatible movement of the commuters. Hence, pedestrian safety is an integral part of overall transport system. Most central business districts are characterized by high intensity development within a small area with resultant congestion and conflicting pedestrian-vehicle movements, especially at signalized intersections. The downtown areas experience highest concentration of day-time population and diverse activities causing vehicle delays, air pollution, and accident hazards. In some cases the conflicting movements of vehicles and pedestrians have been segregated by implementing horizontal, and vertical separation like sidewalks, auto-restricted zones, partial and full malls, underground tunnels, and other innovative approaches. A few cities have diluted the complexity of the problem to some extent by the construction and operation of skywalk systems. A skywalk provides vertical separation of pedestrian and vehicular movements and, along with street level sidewalks, represents a unified system of pedestrian facilities in the central business districts. Pedestrian skywalk is, in practice, very much a transportation system management technique. Pedestrian modelling is a complex behavioral and engineering issue. Of interest to urban transport planners is both the behavioral side i.e., how pedestrians move in relation to other pedestrians, how they decide to cross the road, how they make route choice decisions and the engineering side, i.e., how traffic control measures affect pedestrian travel times [2]. Various modelling approaches have attempted to take into account these aspects, mostly independent of each other. Pedestrian specific micro-simulation techniques have been 
steadily improving over the last decade and have been increasingly applied to crowd movements and building evacuation scenarios with highly sophisticated behavioral algorithms.

With Pedestrian issues considering primarily from the safety point of view rather than Level of Service, amenity. At traffic signals, pedestrians are often accommodated in which least amount of interruption to motorised traffic, and signal cycle times can be long, leading to excessive pedestrian waiting times. This can result to frustration, causing pedestrians to violate the signals and use their own judgment to cross, resulting in safety risks [1]. At traffic signals, pedestrians are often accommodated in a way that causes the least amount of interruption to motorised traffic, and signal cycle times can be long, leading to excessive pedestrian waiting times. This can lead to frustration, causing pedestrians to violate the signals and use their own judgment to cross, resulting in safety risks.

Delay has been widely accepted as a key feature of designing or improving the existing facilities, or to carry out further studies which bring desire changes. Pedestrian delay is an important objective to optimize the effective delay usually carried by pedestrian either because of vehicle hindrance. The service measure is the average delay experienced by a pedestrian this average delay per pedestrian for a crosswalk is given in HCM 2000. Pedestrians obey certain speed-flow and speed-density relationships when pedestrian speeds fall below the desired speed due to higher volumes and densities. Pedestrian speed-flow relationships are well documented in the literature and any multimodal micro-simulation study that includes pedestrian movements should adhere to these relationships. VISSIM offers two different behaviors to model pedestrian flow. The first of these is to state no-interaction between pedestrians. With this constraint all pedestrian movements are independent of the presence of other pedestrians. Any speed-flow relationship is then essentially linear, with speed remaining equal to the desired pedestrian speed irrespective of the pedestrian flow and density. The second possible way to model pedestrian flow is to use the vehicle-following model specified in, which in VISSIM and based on Wiedemann (PTV AG, 2004). This means that pedestrians are defined as vehicles. In that situation pedestrians react to the presence of other pedestrians, under the rules developed for vehicles rather than pedestrians [2].

This paper is organized in four sections including this section. Section two discusses the Criteria for finding pedestrian delay .while section third discusses the Pedestrian microsimulation which is done with the computer application VISSIM 6 software. In section four one combined approach analysis to estimate the pedestrian delay under mixed traffic condition and section five concludes the paper by presenting important conclusions, and pointing the directions for further research.

\section{CRITERIA FOR FINDING PEDESTRIAN} DELAY

Delay has been widely accepted as the key performance index for signalized intersections, thus it is necessary to estimate delays accurately for all kind of traffic participants at signalized intersection. Many research has been conducted for finding vehicle delays at signalized intersection under a broad range of conditions, but eventually research on pedestrian delay is much less. In Highway Capacity Manual (HCM), sophisticated models are provided to estimate vehicle delays at signalized intersection, but the provided pedestrian delay model is quite simple (Transportation Research Board, 2000). At Signalized Intersections

The method of critical gap estimation should be simple and easy to implement. It should be applicable to all traffic conditions and should be generalized enough for its application to mixed as well as uniform traffic and similar it should be work for over saturated as well as under saturated traffic condition. The signalized intersection crossing is more complicated issue to analyse as compare to midblock crossing, because it involves pedestrians crossing the street, intersecting sidewalk flows, and others queued waiting for the signal to change. Research indicates that the average delay for pedestrians at signalized intersection crossings is not constrained by capacity, even when pedestrian flow rates reach $5,000 \mathrm{p} / \mathrm{h}$ [1]. The average delay per pedestrian for a crosswalk is given by:

$$
\mathrm{dp}=\frac{0.5(C-g)^{2}}{C}
$$

Where, $\mathrm{dp}=$ average pedestrian delay $(\mathrm{s}), \mathrm{g}=$ effective green time (for pedestrians) (s), and $\mathrm{C}=$ cycle length(s).

LOS criteria for pedestrians at signalized intersections, based on pedestrian delay. When pedestrians experience more than a 30-s delay, they become impatient, and engage in risk-taking behavior the time. The higher the delay, the poorer is the level of service. The pedestrian delays could be calculated using the 2010 Highway Capacity Manual (HCM 2010) (Transportation Research Board 2010 According to the 2010 HCM method, pedestrian delays while waiting to cross an intersection approach are computed as:

$$
\text { DELAYSPED; } \mathrm{HCM}=\frac{(\mathrm{TG}+\mathrm{TY}+\mathrm{TR}+\mathrm{gWALK})^{2}}{2 \times(\mathrm{TG}+\mathrm{TY}+\mathrm{TR}+4)}
$$

Where DELAYSPED, HCM = Average pedestrian delays per pedestrian per cycle computed using the $2010 \mathrm{HCM}$ method, in $\mathrm{s}=$ ped=cycle; TG, TY, TR = Green, yellow, and red intervals of a cycle, in s; and gWALK = Effective green time for pedestrians to cross an intersection, in $\mathrm{s}$. 


\section{PEDESTRIAN MICROSIMULATION}

Microsimulation traffic models have become widely accepted tools to analyze and identify solutions for vehicle traffic. Pedestrian microsimulation is still in its infancy, research into employing microsimlation for pedestrian analysis has flourished in the past decade. (Teknomo 2002; Daamen 2004; Klugl and Rindsfuser 2007; Kretz 2007) [3]. According to Schoon (2010), use of computerized methods for analysis and design of pedestrian facilities stems from the need to examine extensive amounts of data utilizing complex computational methods and to satisfy required design variables, inputs, and outputs. Among the more widely employed pedestrian circulation software's are VISSIM, SimWalk, Legion and NoMad. VISSIM (PTV-AG) employing the social force model (Helbing and Molnar 1995) pedestrian behavior model was employed to evaluate the effects of pedestrian heterogeneity. VISSIM employs the social force model published by Helbing and Molnar (1995) designed to represent the stochastic behavior of pedestrian movements. Simulation in VISSIM is also the interaction between pedestrians and other traffic and hence how the vehicular traffic is influenced by the pedestrians (PTV Group, 2011). Such a simulation may help to improve current infrastructure in cities where the pedestrian volumes are significantly large. On a more detailed level, it can be helpful when it comes to planning the location of new pedestrian crossings or the width of a sidewalk.

\section{ANALYSIS}

\subsection{Coding of Network}

Microscopic network coding is a time consuming task. Geometric and control attributes are both required. Geometric attributes which include link lengths and widths), number of lanes, link Geometry (shape points) lane connectivity, placement of priority rules and junction layouts. Control attributes include control type (pretimed/ actuated signal, stop/yield signs), phasing and timing plans. VISSIM allows for easy editing of the road network and link properties according to changes in the environment and needs of the study. In VISSIM the road network is a combination of links and connectors. Links are generally straight or follow the curvature of the road. Connectors, which are used to connect links, are typically used to model turning areas and lane expansions and contractions. The network in VISSIM model was constructed by graphical interface and tracing the roadway network over the aerial photographs which served as a background. In VISSIM coding of a road network is not that much easier and faster. Unlike SYNCHRO, in VISSIM it is not sufficient to place one link on top of another link in order for vehicles to continue on the other link. Instead a connector needs to be created to connect the two links. Furthermore connectors are used to model multiple maneuvers (i.e. leftturns, right-turns, U-turns). Each turning movements are required to be coded separately by connectors. The study area includes 2/3lane road stretches (only one direction). By graphical editing of links and connectors, their properties were modified according to field conditions (i.e. no. of lanes, width of lanes, curvature of turning lanes, etc.).

As Income Tax Intersection comes in urban areas being comprise of various institutes along the stretch of road like Nav-Gujarat College, Gujarat Vidyapith. Baba-saheb Open University, etc. Here pedestrian movements across road are high due to many commercial buildings, recreational centers located by the side of road. This the main center area where old Ahmedabad and new Ahmedabad get connected, therefore it becomes most operational route for lane users.

Analysis for the collected data, these are taken in the peak hours of morning and evening for different locations. The selected field area as Income Tax Circle in Ahmedabad

\subsection{Calculation of Field Data}

Field data is being taken as mention above, signal design is being taken to optimize the required green interval time to vehicular traffic and pedestrian traffic. The calculated data is given as in table:

Table -1: Calculation of Signal Timing

\begin{tabular}{|c|c|c|c|c|c|c|c|c|c|c|c|c|}
\hline Leg & & $\operatorname{man} p$ & oura & Ash & hram & raod & Gan & dhi br & igde & & P.Stad & lium \\
\hline Directions & $\mathrm{L}$ & $\mathrm{s}$ & $R$ & $\mathrm{~L}$ & $S$ & $\mathrm{R}$ & $L$ & $S$ & $\mathrm{R}$ & $\mathrm{L}$ & $S$ & $\mathrm{R}$ \\
\hline No. of Veh. & 177 & 947 & 336 & 144 & 709 & 572 & 224 & 1180 & 609 & 129 & 987 & 225 \\
\hline Cr-Left Turn & 44 & & & 36 & & & 56 & & & 32 & & \\
\hline Cr-Right Turn & & & 252 & & & 429 & & & 457 & & & 169 \\
\hline Total & 221 & 947 & 588 & 180 & 709 & 1001 & 280 & 1180 & 1066 & 161 & 987 & 394 \\
\hline Road Width & 3 & 10. & 55 & 3 & & .55 & 3 & 9. & 75 & 3 & 9. & .7 \\
\hline $\mathrm{PCU} / \mathrm{Hr}$ & & 1535 & & & 1710 & & & 2246 & & & 1381 & \\
\hline$S$ & & 3746.6 & & & 12443 & & & 2704. & & & 12639. & \\
\hline$y=q / S$ & 0.1 & 11663 & 5669 & & 13741 & 9487 & & 176771 & 1553 & & .09244 & 4329 \\
\hline$y$ & & & & & & 0.535 & 50989 & & & & & \\
\hline L & & & & & & & 54 & & & & & \\
\hline Co & & & & & & & 85.0 & & & & & \\
\hline Co-L & & & & & & & 31.0 & & & & & \\
\hline Gua & & & & & & & 27 & & & & & \\
\hline Gsg & & & & & & & 27 & & & & & \\
\hline Gau & & & & & & & 34 & & & & & \\
\hline Ggs & & & & & & & 43 & & & & & \\
\hline Ped & & & & & & & 39 & & & & & \\
\hline
\end{tabular}

One of the important step in designing a fixed time signal system is to determine the cycle time. Main consideration in selecting the cycle time should be that the least delay is caused to the traffic passing through the intersection. For total delay for the intersection with respect to cycle time the optimum cycle time as calculated $\mathrm{Co}$ (seconds):

$$
\mathrm{Co}=\frac{1.5 \mathrm{~L}+5}{1-\mathrm{Y}}
$$


$\mathrm{L}=$ total lost time per cycle (sec)

$\mathrm{Y}=\mathrm{y} 1+\mathrm{y} 2+\mathrm{y} 3+\ldots+\mathrm{yn}$... are the max. Ratios of flow to saturation where $\mathrm{q}$ is Flow and $\mathrm{S}$ is Saturation Flow

Saturation flow should be measured rather than estimated value. For designing new signal timings the following simple formula devised as:

$$
\mathrm{S}=1303 \mathrm{w} \mathrm{PCU} / \mathrm{Hr}(6)
$$

Effective Green Time per Cycle $=$ Co-L $(\mathrm{sec})$

The effective green times(seconds)for the phases can be calculated as follows:

G(usmanpura-ashram road)=yua (Co-L)/ Y

$\mathrm{G}($ s.p.stadium-gandhi bridge $)=y s g(\mathrm{Co}-\mathrm{L}) / \mathrm{Y}$

$\mathrm{G}($ ashram road-usmanpura $)=$ yau $(\mathrm{Co}-\mathrm{L}) / \mathrm{Y}$

$\mathrm{G}($ gandhi bridge-s.p.stadium $)=$ ygs $(\mathrm{Co}-\mathrm{L}) / \mathrm{Y}$

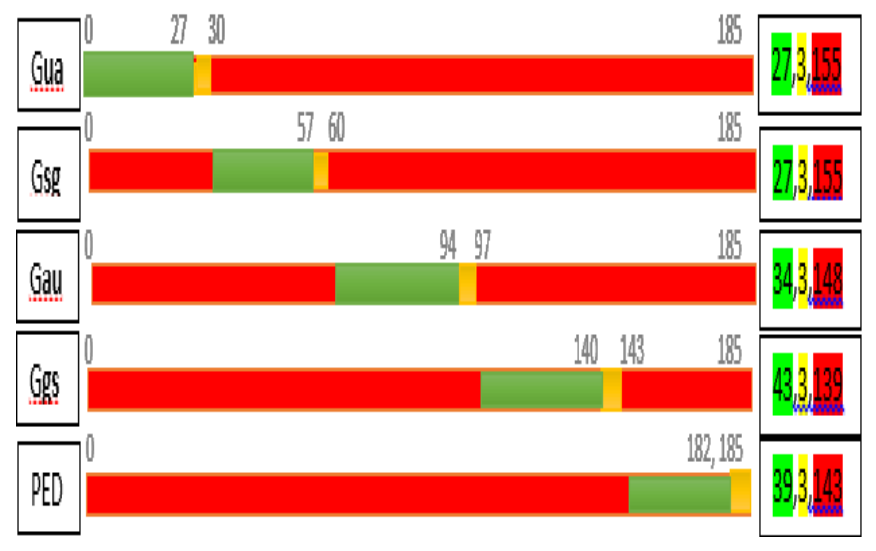

Fig 1-Sketch of Phasing Diagram for each phase(Income Tax Intersection (Ahmedabad).

By analyzing the field data, it gives result in congestion aspect. With the respective cycle time, the average delay per pedestrian comes as $57 \mathrm{sec}$. this facility for pedestrians is not sufficient. Some measure step is to be designed.

\subsection{VISIM Output}

In a delay measurement, the average delay compared to the ideal trip is calculated for all observed vehicles on one or more route sections. As per the data collected for different road stretches, the output of VISSIM is given below:

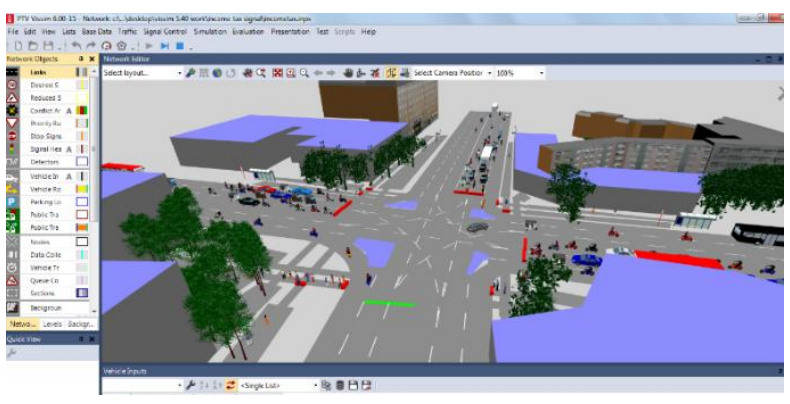

Fig 2-Snap shot of Income Tax Intersection (Ahmedabad) during Simulation run (3D).

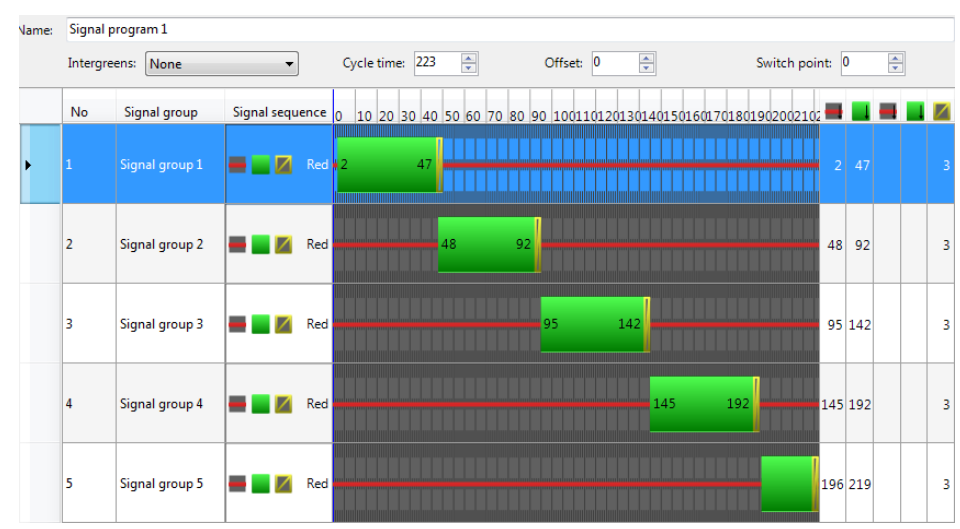

Fig 3-Sketch of Phasing Diagram for each phase (Income Tax Intersection (Ahmedabad). during Simulation in VISSIM

By analyzing the simulated data, it is observed that the traffic leads to congestion the average delay per pedestrian comes 89 sec. By increasing pedestrian phase vehicular traffic will get affected

\subsection{Comparison}

By comparing the field data with the simulated one the result implies that the simulated delay not fairly represent the observed delay.

\section{CONCLUSIONS}

This paper is attempted to obtain an estimated delay with some of the important methods, developed by HCM 2000[5], with their characteristics and suitability to Indian conditions. Many estimations methods are available to estimate the pedestrian delay hence it is difficult to understand which procedure is reliable and which is not. Therefore, we continued with the computer application software (VISSIM 6), to get the actual simulated values. By comparing simulated values with the observed values we come to the solution, either providing pedestrian phase one can design skywalk at junction. According to the IRC:103-2012[4], the control measures usually adopted in such cases is a traffic signal with exclusive pedestrian phase. We have modified this and suggest to carry out the design of skywalk across junction. Because 
while providing cycle length long vehicle and pedestrian both waiting time will exceed Here pedestrian traffic is large therefore suitable walkway is to be design for the safety and convenience.

\section{REFERENCES}

[1] C Vallyon (2011)Reducing Pedestrian delay at traffic signals .

[2] Muhammad M. ISHAQUE (2001) Pedestrian modeling in urban road networks: issues, limitations and opportunities offered by micro-simulation.

[3] Ronald John Galiza and Luis Ferreira (2013) Standard Pedestrian Equivalent Factors: New Approach to Analyzing Pedestrian Flow. Journal of transportation engineering.

[4] IRC 103-2012 Guidelines for Pedestrian Facilities.

[5] Highway Capacity Manual (HCM) 2000.

[6] Momin Safa, H,K Dave International Journal of Engineering and Technical Research (IJETR) Volume1, Issue-1, March 2013 